\title{
A CLT for Plancherel representations of the infinite-dimensional unitary group
}

\author{
Alexei Borodin and Alexey Bufetov
}

\begin{abstract}
We study asymptotics of traces of (noncommutative) monomials formed by images of certain elements of the universal enveloping algebra of the infinitedimensional unitary group in its Plancherel representations. We prove that they converge to (commutative) moments of a Gaussian process that can be viewed as a collection of simply yet nontrivially correlated two-dimensional Gaussian Free Fields. The limiting process has previously arisen via the global scaling limit of spectra for submatrices of Wigner Hermitian random matrices.

This note is an announcement, proofs will appear elsewhere.
\end{abstract}

\section{Introduction}

Asymptotic studies of measures on partitions of representation theoretic origin is a well-known and popular subject. In addition to its intrinsic importance in representation theory, see e.g. 4 and references therein, it enjoys close connections to the theory of random matrices, interacting particle systems, enumerative combinatorics, and other domains, for which it often provides crucial technical tools, cf. e.g. [9], [13].

A typical scenario of how such measures arise is as follows: One starts with a group with a well known list of irreducible representations, often parametrized by partitions or related objects. Then a decomposition of a natural reducible representation of this group on irreducibles provides a split of the total dimension of the representation space into dimensions of the corresponding isotypical components; their relative sizes are the weights of the measure. This procedure is well-defined for finite-dimensional representations, but also for infinite-dimensional representations with finite trace; the weight of (the label of) an isotypical component is then defined as the trace of the projection operator onto it, provided that the trace is normalized to be equal to 1 on the identity operator.

An alternative approach to measures of this sort consists in defining averages with respect to such a measure for a suitable set of functions on labels of the irreducible representations. These averages are obtained as traces of the operators in the ambient representation space that are scalar in each of the isotypical components. In their turn, the operators are images of central elements in the group 
algebra of the group if the group is finite, or in the universal enveloping algebra of the Lie algebra if one deals with a Lie group. The central elements form a commutative algebra that is being mapped to the algebra of functions on the labels, i.e., on partitions or their relatives. The value of the function corresponding to a central element at a representation label is the (scalar) value of this element in that representation.

While one may be perfectly satisfied with such an approach from probabilistic point of view, from representation theoretic point of view it is somewhat unsettling that we are able to only deal with commutative subalgebras this way, while the main interest of representation theory is in noncommutative effects.

The goal of this work is go beyond this commutativity constraint.

More exactly, in a specific setting of the finite trace representations of the infinite-dimensional unitary group described below, we consider a family of commutative subalgebras of the universal enveloping algebra such that elements from different subalgebras generally speaking do not commute. We further consider the limit regime in which the measures for each of the commutative subalgebras are known to approximate the two-dimensional Gaussian Free Field (GFF), see [3]. We want to study the "joint distribution" of these GFFs for different subalgebras, whatever this might mean.

For any element of the universal enveloping algebra, one can define its "average" as the trace of its image in the representation. Thus, having a representation, we can define "averages" for arbitrary products of elements from our subalgebras, despite the fact that the elements do not commute.

Our main result is that for certain Plancherel representations, these "averages" converge to actual averages of suitable observables on a Gaussian process that consists of a family of explicitly correlated GFFs. Thus, the original absence of commutativity in this limit disappears, and yet the limiting GFFs that arise from different commutative subalgebras do not become independent.

The same limiting object (the collection of correlated GFFs) has been previously shown to be the universal global scaling limit for eigenvalues of various submatrices of Wigner Hermitian random matrices, cf. [2]. We also expect it to arise from other, non-Plancherel factor representations of the infinite-dimensional unitary group under appropriate limit transitions.

The present paper is an announcement, the proofs will appear in a subsequent publication.

\section{Acknowledgements}

The authors are very grateful to Grigori Olshanski for numerous discussions that were extremely helpful. A. Borodin was partially supported by NSF grant DMS1056390. A. Bufetov was partially supported by Simons Foundation-IUM scholarship, by Moebius Foundation for Young Scientists, and by RFBR-CNRS grant 10-01-93114. 


\section{Characters of unitary groups}

Let $I$ be a finite set of natural numbers, and let $U(I)=\left(u_{i j}\right)_{i, j \in I}$ be the group of unitary matrices whose rows and columns are marked by elements of $I$. In what follows we denote $\{1,2, \ldots, N\}$ as $\overline{1, N}$. Consider the tower of embedded unitary groups

$$
U(\{1\}) \subset U(\{1,2\}) \subset \ldots U(\overline{1, N}) \subset U(\overline{1, N+1}) \subset \ldots,
$$

where the embedding $U(\overline{1, k}) \subset U(\overline{1, k+1})$ is defined by $u_{i, k+1}=u_{k+1, i}=0$, $1 \leq i \leq k, u_{k+1, k+1}=1$. The infinite-dimensional unitary group is the union of these groups:

$$
U(\infty)=\bigcup_{N=1}^{\infty} U(\overline{1, N}) .
$$

A signature (also called highest weight) of length $N$ is a sequence of $N$ weakly decreasing integers $\lambda_{1} \geq \lambda_{2} \geq \cdots \geq \lambda_{N}$. Let $\mathbb{G T}_{N}$ denote the set of such signatures. (Here the letters $\mathbb{G} \mathbb{T}$ stand for 'Gelfand-Tsetlin'.) We say that $\lambda \in \mathbb{G T}_{N}$ and $\mu \in \mathbb{G T}_{N-1}$ interlace, notation $\mu \prec \lambda$, iff $\lambda_{i} \geq \mu_{i} \geq \lambda_{i+1}$ for any $1 \leq i \leq N-1$. We also define $\mathbb{G T}_{0}$ as a singleton consisting of an element that we denote as $\varnothing$. We assume that $\varnothing \prec \lambda$ for any $\lambda \in \mathbb{G}_{1}$.

The Gelfand-Tsetlin graph $\mathbb{G} \mathbb{T}$ is defined by specifying its set of vertices as $\bigcup_{N=0}^{\infty} \mathbb{G T}_{N}$ and putting an edge between any two signatures $\lambda$ and $\mu$ such that either $\lambda \prec \mu$ or $\mu \prec \lambda$. A path between signatures $\kappa \in \mathbb{G T}_{K}$ and $\nu \in \mathbb{G T}_{N}, K<N$ is a sequence

$$
\kappa=\lambda^{(K)} \prec \lambda^{(K+1)} \prec \cdots \prec \lambda^{(N)}=\nu, \quad \lambda^{(i)} \in \mathbb{G}_{i} .
$$

Let $\operatorname{Dim}_{N}(\nu)$ be the number of paths between $\varnothing$ and $\nu \in \mathbb{G T}_{N}$. An infinite path is a sequence

$$
\varnothing \prec \lambda^{(1)} \prec \lambda^{(2)} \prec \cdots \prec \lambda^{(k)} \prec \lambda^{(k+1)} \prec \ldots
$$

We denote by $\mathcal{P}$ the set of all such paths. It is a topological space with the topology induced from the product topology on the ambient product of discrete sets $\prod_{N>0} \mathbb{G T}_{N}$.

For $\bar{N}=0,1,2, \ldots$, let $M_{N}$ be a probability measure on $\mathbb{G T}_{N}$. We say that $\left\{M_{N}\right\}_{N=0}^{\infty}$ is a coherent system of measures if for any $N \geq 0$ and $\lambda \in \mathbb{G}_{N}$,

$$
M_{N}(\lambda)=\sum_{\nu: \lambda \prec \nu} M_{N+1}(\nu) \frac{\operatorname{Dim}_{N}(\lambda)}{\operatorname{Dim}_{N}(\nu)}
$$

Given a coherent system of measures $\left\{M_{n}\right\}_{n=1}^{\infty}$, define a weight of a cylindric set of $\mathcal{P}$ consisting of all paths with prescribed members up to $\mathbb{G T}_{N}$ by

$$
P\left(\lambda^{(1)}, \lambda^{(2)}, \ldots, \lambda^{(N)}\right)=\frac{M_{N}\left(\lambda^{(N)}\right)}{\operatorname{Dim}_{N}\left(\lambda^{(N)}\right)} .
$$


Note that this weight depends on $\lambda^{(N)}$ only. The coherency property implies that these weights are consistent, and they correctly define a Borel probability measure on $\mathcal{P}$.

It is well known that the irreducible (complex) representations of $U(N)=$ $U(\overline{1, N})$ can be parametrized by signatures of length $N$, and $\operatorname{Dim}_{N}(\lambda)$ is the dimension of the representation corresponding to $\lambda$. Let $\chi^{\lambda}$ be the conventional character of this representation (i.e., the function on the group obtained by evaluating trace of the representation operators) divided by $\operatorname{Dim}_{N}(\lambda)$.

Define a character of the group $U(\infty)$ as a function $\chi: U(\infty) \rightarrow \mathbb{C}$ that satisfies

1) $\chi(e)=1$, where $e$ is the identity element of $U(\infty)$ (normalization);

2) $\chi\left(g h g^{-1}\right)=\chi(h)$, where $g, h$ are any elements of $U(\infty)$ (centrality);

3) $\chi\left(g_{i} g_{j}^{-1}\right)_{i, j=1}^{n}$ is an Hermitian and positive-definite matrix for any $n \geq 1$ and $g_{1}, \ldots, g_{n} \in U(\infty)$ (positive-definiteness);

4) the restriction of $\chi$ to $U(\overline{1, N})$ is a continuous function for any $N \geq 1$ (continuity).

Let $\chi$ be a character of $U(\infty)$. It turns out that for any $N \geq 1$, its restriction to $U(N)$ can be decomposed into a series in $\chi^{\lambda}$,

$$
\left.\chi\right|_{U(N)}=\sum_{\lambda \in \mathbb{G T}_{N}} M_{N}(\lambda) \chi^{\lambda},
$$

and the coefficients $M_{N}(\lambda)$ form a coherent system of measures on $\mathbb{G} \mathbb{T}$. Conversely, for any coherent system of measures on $\mathbb{G} \mathbb{T}$ one can construct a character of $U(\infty)$ using the above formula.

The space of characters of $U(\infty)$ is obviously convex. The extreme points of this set can be viewed as traces of the factor-representations of $U(\infty)$ with finite trace, or as spherical functions for irreducible spherical unitary representations of the Gelfand pair $(U(\infty) \times U(\infty), \operatorname{diag}(U(\infty)))$, see 12 for details. The classification of the extreme characters is known as Edrei-Voiculescu theorem, see [10] and references therein.

\section{Characters and states on the universal envelop- ing algebra}

Let $\mathfrak{g l}(I)=\left(g_{i j}\right)_{i, j \in I}$ be the complexified Lie algebra of $U(I)$, let $\mathcal{U}(\mathfrak{g l}(I))$ be its universal enveloping algebra, and let $Z(\mathfrak{g l}(I))$ be the center of $\mathcal{U}(\mathfrak{g l}(I))$. Denote by

$$
\mathcal{U}(\mathfrak{g l}(\infty))=\bigcup_{N \geq 1} \mathcal{U}(\mathfrak{g l}(\overline{1, N}))
$$

the universal enveloping algebra of $\mathfrak{g l}(\infty)$.

There exists a canonical isomorphism

$$
D_{I}: \mathcal{U}(\mathfrak{g l}(I)) \rightarrow \mathcal{D}(I)
$$


where $\mathcal{D}(I)$ is the algebra of left-invariant differential operators on $U(I)$ with complex coefficients. Let $\left\{x_{i j}\right\}$ be the matrix coordinates. For any character $\chi$ define a state on $\mathcal{U}(\mathfrak{g l}(\infty))$ as follows: For any $X \in \mathcal{U}(\mathfrak{g l}(\infty))$

$$
\langle X\rangle_{\chi}=\left.D_{I}(X) \chi\left(x_{i j}\right)\right|_{x_{i j}=\delta_{i j}}, \quad X \in \mathcal{U}(\mathfrak{g l}(I)) .
$$

Note that this definition is consistent for different choices of $I$.

We shall denote coordinates of the signatures that parametrize irreducible representations of $U(I)$ as $\lambda_{1}^{(I)}, \ldots, \lambda_{|I|}^{(I)}$. There exists a canonical isomorphism $Z(\mathfrak{g l}(I)) \rightarrow \mathbb{A}(I)$, where $\mathbb{A}(I)$ is the algebra of shifted symmetric polynomials in $\lambda_{1}^{(I)}, \ldots, \lambda_{|I|}^{(I)}$, see e.g. [11]. For any central element, the value of the corresponding function at a signature corresponds to the (scalar) operator that this element turns into in the corresponding representation.

Similarly to Section 2, restricting $\chi$ to $U(I)$ gives rise to a probability measure on signatures of length $|I|$. One shows that the state $\langle\cdot\rangle_{\chi}$ on an element of $Z(\mathfrak{g l}(I))$ equals the expectation of the corresponding function in $\mathbb{A}(I)$ with respect to this probability measure.

One can also evaluate $\langle\cdot\rangle_{\chi}$ as an expectation on a larger probability space. Consider $\{1\} \subset\{1,2\} \subset \ldots \subset \overline{1, k} \subset \ldots$ Let $\mathcal{Z}$ be the subalgebra in $\mathcal{U}(\mathfrak{g l}(\infty))$ generated by all the centers $Z(\mathfrak{g l}(\overline{1, k})), k=1,2, \ldots$ To any element of $Z(\mathfrak{g l}(\overline{1, k}))$ we assign a function on the path space $\mathcal{P}$ by taking the length $k$ member of the path and applying the isomorphism $Z(\mathfrak{g r}(\overline{1, k})) \rightarrow \mathbb{A}(\overline{1, k})$. Hence, the algebra $\mathcal{Z}$ is naturally embedded into functions on $\mathcal{P}$. Denote the probability measure on $\mathcal{P}$ that arises from the coherent system of measures originated from $\chi$ by $\mu_{\chi}$. Then the value of $\langle\cdot\rangle_{\chi}$ on any element of $\mathcal{Z}$ is equal to the expectation of the corresponding function on the probability space $\left(\mathcal{P}, \mu_{\chi}\right)$.

\section{One-sided Plancherel characters}

In what follows we restrict ourselves to the one-sided Plancherel character with a growing parameter. This extreme character is defined as

$$
\chi(U)=\exp \left(\gamma L \sum_{i=1}^{\infty}\left(x_{i i}-1\right)\right),
$$

where $U=\left[x_{i j}\right]_{i, j \geq 1}, \gamma$ is fixed, and $L$ is the growing parameter. Denote by $\mu_{\gamma}$ the probability measure on $\mathcal{P}$ that corresponds to this character (cf. Section 2), and denote by $\langle\cdot\rangle_{\gamma}$ the state that corresponds to this character (cf. Section 3 ).

If one restricts (3) to $U(I)$ and decomposes it on normalized conventional characters of $U(I)$, one obtains a probability measure on signatures of length $|I|$ of the form

$$
P_{I}^{\gamma L}(\lambda):= \begin{cases}e^{-\gamma L|I|} \frac{(\gamma L)^{\lambda_{1}+\cdots+\lambda_{|I|}}}{\left(\lambda_{1}+\cdots+\lambda_{|I|}\right) !} \operatorname{dim} \lambda \operatorname{Dim}_{|I|} \lambda, & \lambda_{1} \geq \cdots \geq \lambda_{|I|} \geq 0 \\ 0, & \text { otherwise }\end{cases}
$$


where $\operatorname{dim} \lambda$ is the dimension of the irreducible representation of the symmetric group $S_{|\lambda|}$ that corresponds to $\lambda$ (= the number of standard Young tableaux of shape $\lambda$ ). Observe that these probability measures are supported by nonnegative signatures, i.e., on partitions or Young diagrams.

Asymptotic properties of $P \frac{\gamma L}{1, L}$ as $L \rightarrow \infty$ and related distributions have been extensively studied in [1], [3], [5], [7, [8].

\section{Random height functions and GFF}

A Gaussian family is a collection of Gaussian random variable $\left\{\xi_{a}\right\}_{a \in \Upsilon}$ indexed by an arbitrary set $\Upsilon$. We assume that all the random variable are centered, i.e.

$$
\mathbf{E} \xi_{a}=0, \quad \text { for any } a \in \Upsilon .
$$

Any Gaussian family gives rise to the covariance kernel Cov $: \Upsilon \times \Upsilon \rightarrow \mathbb{R}$ defined (in the centered case) by

$$
\operatorname{Cov}\left(a_{1}, a_{2}\right)=\mathbf{E}\left(\xi_{a_{1}} \xi_{a_{2}}\right) .
$$

Assume that a function $\tilde{C}: \Upsilon \times \Upsilon \rightarrow \mathbb{R}$ is such that for any $n \geq 1$ and $a_{1}, \ldots, a_{n} \in \Upsilon,\left[\tilde{C}\left(a_{i}, a_{j}\right)\right]_{i, j=1}^{n}$ is a symmetric and positive-definite matrix. Then (see e.g. 6]) there exists a centered Gaussian family with the covariance function $\tilde{C}$.

Let $\mathbb{H}:=\{z \in \mathbb{C}: \Im(z)>0\}$ be the upper half-plane, and let $C_{0}^{\infty}$ be the space of smooth real-valued compactly supported test functions on $\mathbb{H}$. Define a function $C: C_{0}^{\infty} \times C_{0}^{\infty} \rightarrow \mathbb{R}$ via

$$
C\left(f_{1}, f_{2}\right):=\int_{\mathbb{H}} \int_{\mathbb{H}} f_{1}(z) f_{2}(w)\left(-\frac{1}{2 \pi} \ln \frac{z-w}{z-\bar{w}}\right) d z d \bar{z} d w d \bar{w} .
$$

The Gaussian Free Field (GFF) $\mathfrak{G}$ on $\mathbb{H}$ with zero boundary conditions can be defined as a Gaussian family $\left\{\xi_{f}\right\}_{f \in C_{0}^{\infty}}$ with covariance kernel $C$. The field $\mathfrak{G}$ cannot be defined as a random function on $\mathbb{H}$, but one can make sense of the integrals $\int f(z) \mathfrak{G}(z) d z$ over smooth finite contours in $\mathbb{H}$ with continuous functions $f(z)$, cf. [14.

Define the height function

$$
H: \mathbb{R}_{\geq 0} \times \mathbb{R}_{\geq 1} \times \mathcal{P} \rightarrow \mathbb{N}
$$

as

$$
H(x, y)=\sqrt{\pi}\left|\left\{i \in \overline{1,[y]}: \lambda_{i}^{(y)}-i+1 / 2 \geq x\right\}\right|,
$$

where $\lambda_{i}^{(y)}$ are the coordinates of the signature of length $[y]$ from the infinite path. If we equip $\mathcal{P}$ with a probability measure $\mu_{\gamma}$ then $H(x, y)$ becomes a random function describing a certain random stepped surface, or a random lozenge tiling of the half-plane, see [3]. 
Define $x(z), y(z): \mathbb{H} \rightarrow \mathbb{R}$ via

$$
x(z)=\gamma(1-2 \mathfrak{R}(z)), \quad y(z)=\gamma|z|^{2} .
$$

Let us carry $H(x, y)$ over to $\mathbb{H}$ - define

$$
H^{\Omega}(z)=H(L x(z), L y(z)), \quad z \in \mathbb{H} .
$$

It is known, cf. [1], 3], that there exists a limiting (nonrandom) height function

$$
\tilde{h}(z):=\lim _{L \rightarrow \infty} \frac{\mathbf{E} H^{\Omega}(z)}{L}, \quad z \in \mathbb{H},
$$

that describes the limit shape. The fluctuations around the limit shape were studied in [3], where it was shown that the fluctuation field

$$
\mathcal{H}(z):=H^{\Omega}(z)-\mathbf{E} H^{\Omega}(z), \quad z \in \mathbb{H},
$$

converges to the GFF introduced above.

In [3], this convergence was proved for a certain space of test functions. Let us formulate a similar statement that we prove in this work, and that utilizes a different space of test functions.

Define a moment of the random height function via

$$
M_{y, k}:=\int_{-\infty}^{\infty} x^{k}(H(L x, L y)-\mathbf{E} H(L x, L y)) d x
$$

Also define the corresponding moment of the GFF as

$$
\mathcal{M}_{y, k}=\int_{z \in \mathbb{H} ; y=\gamma|z|^{2}} x(z)^{k} \mathfrak{G}(z) \frac{d x(z)}{d z} d z .
$$

Proposition 1. As $L \rightarrow \infty$, the collection of random variables $\left\{M_{y, k}\right\}_{y>0, k \in \mathbb{Z}_{\geq 0}}$ converges, in the sense of finite dimensional distributions, to $\left\{\mathcal{M}_{y, k}\right\}_{y>0, k \in \mathbb{Z}_{\geq 0}}$.

\section{Convergence in the sense of states}

Consider a probability space $\Omega$ and a sequence of $k$-dimensional random variables $\left(\eta_{n}^{1}, \eta_{n}^{2}, \ldots, \eta_{n}^{k}\right)_{n \geq 1}$ on it that converge, in the sense of convergence of moments, to a Gaussian random vector $\left(\eta^{1}, \ldots, \eta^{k}\right)$ with zero mean. If we define a state as

$$
\langle\xi\rangle_{\Omega}:=\mathbf{E} \xi, \quad \xi \in L^{1}(\Omega),
$$

then this convergence can be reformulated as

$$
\begin{aligned}
& \left\langle\eta_{n}^{i_{1}} \eta_{n}^{i_{2}} \ldots \eta_{n}^{i_{l}}\right\rangle_{\Omega} \underset{n \rightarrow \infty}{\longrightarrow} \sum_{\sigma \in \mathcal{P} \mathcal{M}} \prod_{j=1}^{l / 2}\left\langle\eta^{\sigma(2 j-1)} \eta^{\sigma(2 j)}\right\rangle_{\Omega}, \\
& \qquad \text { for any } l \geq 1 \text { and any }\left(i_{1}, \ldots, i_{l}\right) \in\{1, \ldots, k\}^{l},
\end{aligned}
$$


where $\mathcal{P} \mathcal{M}$ is the set of involutions on $\{1,2, \ldots, l\}$ with no fixed points, also known as perfect matchings. (In particular, $\mathcal{P} \mathcal{M}$ is empty if $l$ is odd). Indeed, Wick's formula implies that the right-hand side of (6) contains the moments of $\eta$.

Let $\mathcal{A}$ be a $*$-algebra and $\langle\cdot\rangle$ be a state (=linear functional taking nonnegative values at elements of the form $\left.a a^{*}\right)$ on it. Let $a_{1}, a_{2}, \ldots, a_{k} \in \mathcal{A}$.

Assume that the elements $a_{1}, \ldots, a_{k}$ and the state on $\mathcal{A}$ depend on a large parameter $L$, and we also have a $*$-algebra $\mathbf{A}$ generated by elements $\mathbf{a}_{1}, \ldots, \mathbf{a}_{k}$ and a state $\phi$ on it. We say that $\left(a_{1}, \ldots, a_{k}\right)$ converge to $\left(\mathbf{a}_{1}, \ldots, \mathbf{a}_{k}\right)$ in the sense of states if

$$
\left\langle a_{i_{1}} a_{i_{2}} \ldots a_{i_{l}}\right\rangle \underset{L \rightarrow \infty}{\longrightarrow} \phi\left(\mathbf{a}_{i_{1}} \ldots \mathbf{a}_{i_{l}}\right),
$$

and this holds for any $l \in \mathbb{N}$ and any index sets $\left(i_{1}, i_{2}, \ldots, i_{l}\right) \in\{1,2, \ldots, k\}^{l}$.

We say that a collection $\left\{a_{i}\right\}_{i \in \mathfrak{J}} \subset \mathcal{A}$ indexed by an arbitrary set $\mathfrak{J}$ and depending on a large parameter $L$, converges in the sense of states to a collection $\left\{\mathbf{a}_{i}\right\}_{i \in \mathfrak{J}} \subset \mathbf{A}$ if (7) holds for any finite subsets of $\left\{a_{i}\right\}_{i \in \mathfrak{J}}$ and corresponding subsets in $\left\{\mathbf{a}_{i}\right\}_{i \in \mathfrak{J}}$.

In what follows, the algebra $\mathcal{A}$ is taken to be $\mathcal{U}(\mathfrak{g l}(\infty))$, and the state is taken to be $\langle\cdot\rangle_{\gamma}$, cf. (2), (3). The role of the limiting algebra $\mathbf{A}$ will be played by a commutative algebra originated from a probability space.

\section{The main result}

Let $A=\left\{a_{n}\right\}_{n \geq 1}$ be a sequence of pairwise distinct natural numbers. Let $\mathcal{P}_{A}$ be a copy of the path space $\mathcal{P}$ corresponding to $A$. Given $A$, we define the height function

$$
\begin{gathered}
H_{A}: \mathbb{R}_{\geq 0} \times \mathbb{R}_{\geq 1} \times \mathcal{P}_{A} \rightarrow \mathbb{N} \\
H_{A}(x, y)=\sqrt{\pi}\left|\left\{i \in \overline{1,[y]}: \lambda_{i}^{\left(\left\{a_{1}, \ldots, a_{[y]}\right\}\right)}-i+1 / 2 \geq x\right\}\right|,
\end{gathered}
$$

where $\lambda_{i}^{\left(\left\{a_{1}, \ldots, a_{[y]}\right\}\right)}$ are the coordinates of the length $[y]$ signature in the infinite path. Under the probability measure $\mu_{\gamma}$ on $\mathcal{P}_{A}, H_{A}(x, y)$ becomes a random function on the probability space $\left(\mathcal{P}_{A}, \mu_{\gamma}\right)$.

Let $\left\{A_{i}\right\}_{i \in \mathfrak{J}}$ be a family of sequences of pairwise distinct natural numbers, indexed by a set $\mathfrak{J}$. Introduce the notation

$$
A_{i}=\left\{a_{i, n}\right\}_{n \geq 1}, \quad A_{i, m}=\left\{a_{i, 1}, \ldots, a_{i, m}\right\} .
$$

The coordinates $a_{i, j}=a_{i, j}(L)$ may depend on the large parameter $L$. a limit

We say that $\left\{A_{i}\right\}_{i \in \mathfrak{J}}$ is regular if for any $i, j \in \mathfrak{J}$ and any $x, y>0$ there exists

$$
\alpha(i, x ; j, y)=\lim _{L \rightarrow \infty} \frac{\left|A_{i,[x L]} \cap A_{j,[y L]}\right|}{L} .
$$


For example, the following family is regular: $\mathfrak{J}=\{1,2,3,4\}$ with $a_{1, n}=n$, $a_{2, n}=2 n, a_{3, n}=2 n+1$, and

$$
a_{4, n}= \begin{cases}n+L, & n=1,2, \ldots, L, \\ n-L, & n=L+1, L+2, \ldots, 2 L, \\ n, & n \geq 2 L+1 .\end{cases}
$$

Consider the union of copies of $\mathbb{H}$ indexed by $\mathfrak{J}$ :

$$
\mathbb{H}(\mathfrak{I}):=\bigcup_{i \in \mathfrak{I}} \mathbb{H}_{i}
$$

Define a function $C: \mathbb{H}(\mathfrak{J}) \times \mathbb{H}(\mathfrak{J}) \rightarrow \mathbb{R} \cup\{+\infty\}$ via

$$
C_{i j}(z, w)=\frac{1}{2 \pi} \ln \left|\frac{\alpha\left(i,|z|^{2} ; j,|w|^{2}\right)-z w}{\alpha\left(i,|z|^{2} ; j,|w|^{2}\right)-z \bar{w}}\right|, \quad i, j \in \mathfrak{J}, \quad z \in \mathbb{H}_{i}, w \in \mathbb{H}_{j}
$$

Proposition 2. For any regular family as above, there exists a generalized Gaussian process on $\mathbb{H}(\mathfrak{J})$ with the covariance kernel $C_{i j}(z, w)$. More exactly, for any finite set of test functions $f_{m}(z) \in C_{0}^{\infty}\left(\mathbb{H}_{i_{m}}\right)$ and $i_{1}, \ldots, i_{M} \in \mathfrak{J}$, the covariance matrix

$$
\operatorname{cov}\left(f_{k}, f_{l}\right)=\int_{\mathbb{H}} \int_{\mathbb{H}} f_{k}(z) f_{l}(w) C_{i_{k} i_{l}}(z, w) d z d \bar{z} d w d \bar{w}
$$

is positive-definite.

Proof. See [2, Proposition 1].

Let us denote this Gaussian process as $\mathfrak{G}_{\left\{A_{i}\right\}_{i \in \mathfrak{J}}}$. Its restriction to a single half-plane $\mathbb{H}_{i}$ is the GFF introduced above because

$$
C_{i i}(z, w)=-\frac{1}{2 \pi} \ln \left|\frac{z-w}{z-\bar{w}}\right|, \quad z, w \in \mathbb{H}_{i}, \quad i \in \mathfrak{J} .
$$

As before, let us carry $H_{A}(x, y)$ over to $\mathbb{H}$ - define

$$
H_{A}^{\Omega}(z)=H_{A}(L x(z), L y(z)), \quad z \in \mathbb{H} .
$$

As was mentioned above, the fluctuations

$$
\mathcal{H}_{i}(z):=H_{A_{i}}^{\Omega}(z)-\mathbf{E} H_{A_{i}}^{\Omega}(z), \quad i \in \mathfrak{J}, z \in \mathbb{H}_{i}
$$

for any fixed $i$ converge to the GFF.

The main goal of this paper is to study the joint fluctuations (10) for different $i$. The joint fluctuations of $\mathcal{H}_{i}$ are understood as follows. Define the moments of the random height function as

$$
M_{i, y, k}:=\int_{-\infty}^{\infty} x^{k}\left(H_{A_{i}}(L x, L y)-\mathbf{E} H_{A_{i}}(L x, L y)\right) d x .
$$


It turns out that the function $M_{i, y, k}$ belongs to $\mathbb{A}\left(A_{i,[L y]}\right)$, and thus it corresponds to an element of $Z\left(\mathfrak{g l}\left(A_{i,[L y]}\right)\right)$; denote this element by the same symbol. Note that all such elements $M_{i, y, k}$ for all $i, y, k$ belong to the ambient algebra $\mathcal{U}(\mathfrak{g l}(\infty))$, and we also have the state $\langle\cdot\rangle_{\gamma}$ defined on this ambient algebra. Thus, we can talk about convergence of such elements in the sense of states, see Section 6. We are interested in the limit as $L \rightarrow \infty$.

We prove that the family $\left\{\mathcal{H}_{i}\right\}_{i \in \mathfrak{J}}$ converges to the generalized Gaussian process $\mathfrak{G}_{\left\{A_{i}\right\}_{i \in \mathfrak{J}}}$. Define the moments of $\mathfrak{G}_{\left\{A_{i}\right\}_{i \in \mathfrak{J}}}$ by

$$
\mathcal{M}_{i, y, k}=\int_{z \in \mathbb{H} ; y=\gamma|z|^{2}} x(z)^{k} \mathfrak{G}_{A_{i}}(z) \frac{d x(z)}{d z} d z .
$$

Theorem 1. As $L \rightarrow \infty$, the moments $\left\{M_{i, y, k}\right\}_{i \in \mathfrak{J}, y>0, k \in \mathbb{Z}_{\geq 0}}$ converge in the sense of states to the moments $\left\{\mathcal{M}_{i, y, k}\right\}_{i \in \mathfrak{J}, y>0, k \in \mathbb{Z}_{\geq 0}}$.

Thus, in the $L \rightarrow \infty$ limit, the noncommutativity disappears (limiting algebra A is commutative), and yet the random fields $\mathcal{H}_{i}$ for different $i$ 's are not independent.

Let $u=L x$. The definition of the height function implies

$$
\frac{d}{d u} H_{A_{i}}(u,[L y])=-\sqrt{\pi} \sum_{s=1}^{[L y]} \delta\left(u-\left(\lambda_{s}^{\left(A_{i,[L y]}\right)}-s+1 / 2\right)\right)
$$

Define the shifted power sums

$$
p_{k, I}=\sum_{i=1}^{|I|}\left(\left(\lambda_{i}^{(I)}-i+\frac{1}{2}\right)^{k}-\left(-i+\frac{1}{2}\right)^{k}\right), \quad I \subset \mathbb{N} .
$$

One shows that $p_{k, I} \in \mathbb{A}(I)$, and hence they correspond to certain elements of $Z(\mathfrak{g l}(I))$ that we will denote by the same symbol.

Integrating (11) by parts shows that $M_{i, y, k}$ can be rewritten as

$$
\begin{array}{r}
\frac{L^{-(k+1)} \sqrt{\pi}}{k+1}\left(\sum_{s=1}^{[L y]}\left(\lambda_{s}^{\left(A_{i,[L y]}\right)}-s+\frac{1}{2}\right)^{k+1}-\mathbf{E} \sum_{s=1}^{[L y]}\left(\lambda_{s}^{\left(A_{i,[L y]}\right)}-s+\frac{1}{2}\right)^{k+1}\right) \\
=\frac{L^{-(k+1) \sqrt{\pi}}}{k+1}\left(p_{k+1, I}-\mathbf{E} p_{k+1, I}\right) .
\end{array}
$$

Thus, Theorem 1 can be reformulated as follows.

Theorem 2. Let $k_{1}, \ldots, k_{m} \geq 1$ and $I_{1}, \ldots, I_{m}$ be finite subsets of $\mathbb{N}$ that may depend on the large parameter $L$ in such a way that there exist limits

$$
\eta_{r}=\lim _{L \rightarrow \infty} \frac{\left|I_{r}\right|}{L}>0, \quad c_{r s}=\lim _{L \rightarrow \infty} \frac{\left|I_{r} \cap I_{s}\right|}{L} .
$$


Then as $L \rightarrow \infty$, the collection

$$
\left(L^{-k_{r}}\left(p_{k_{r}, I_{r}}-\mathbf{E} p_{k_{r}, I_{r}}\right)\right)_{r=1}^{m}
$$

of elements of $\mathcal{U}(\mathfrak{g l}(\infty))$ converges in the sense of states, cf. (7), to the Gaussian vector $\left(\xi_{1}, \ldots, \xi_{m}\right)$ with zero mean and covariance

$$
\begin{aligned}
\mathbf{E} \xi_{r} \xi_{s}=\frac{k_{r} k_{s}}{\pi} \oint_{|z|^{2}=\frac{\eta_{r}}{\gamma} ; \mathfrak{I}(z)>0} \oint_{|w|^{2}=} & \frac{\eta_{s}}{\gamma} ; \mathfrak{I}(w)>0 \\
& \times \frac{1}{2 \pi} \ln \left|\frac{c_{r s} / \gamma-z w}{c_{r s} / \gamma-z \bar{w}}\right| \frac{d(x(z))}{d z} \frac{d(x(w))}{d w} d z d w .
\end{aligned}
$$

\section{References}

[1] P. Biane. Approximate factorization and concentration for characters of symmetric groups. Internat. Math. Res. Notices 2001, no. 4, 179-192.

[2] A. Borodin. CLT for spectra of submatrices of Wigner random matrices, Preprint, 2010, arXiv: 1010.0898

[3] A. Borodin, P.L. Ferrari. Anisotropic growth of random surfaces in $2+1$ dimensions , Preprint, 2008, arXiv:0804.3035.

[4] A. Borodin, G. Olshanski. Representation theory and random point processes. European Congress of Mathematics, 73-94, Eur. Math. Soc., Zürich, 2005, arXiv:math/0409333.

[5] A. Borodin, G. Olshanski. Asymptotics of Plancherel-type random partitions. Journal of Algebra 313 (2007), no. 1, 40-60, arXiv:math/0610240

[6] P. Cartier. Introduction a l'etude des mouvements browniens a plusieurs parametres. Seminaire de probabilites (Strasbourg) 5 (1971), 58-75.

[7] P.L. Méliot. Kerov's central limit theorem for Schur-Weyl measures of parameter 1/2, Preprint, 2010, arXiv:1009.4034

[8] S. Mkrtchyan. Entropy of Schur-Weyl Measures. Preprint, 2011, arXiv:1107.1541

[9] A. Okounkov. The uses of random partitions. XIVth International Congress on Mathematical Physics, 379-403, World Sci. Publ., Hackensack, NJ, 2005, arXiv:math-ph/0309015.

[10] A. Okounkov, G. Olshanski. Asymptotics of Jack polynomials as the number of variables goes to infinity. Intern. Math. Research Notices 1998, no. 13, 641-682, arXiv:q-alg/9709011.

[11] A. Okounkov, G. Olshanski. Shifted Schur functions. Algebra i Analiz 9 (1997), no. 2, 73-146, arXiv:q-alg/9605042.

[12] G. Olshanski. Unitary representations of infinite-dimensional pairs $(G, K)$ and the formalism of $R$. Howe. In: Representations of Lie groups and related topics. Advances in Contemp. Math., vol. 7 (A. M. Vershik and D. P. Zhelobenko, editors). Gordon and Breach, N.Y., London etc. 1990, 269-463. 
[13] G. Olshanski. Random permutations and related topics. Chapter 25 of The Oxford Handbook of Random Matrix Theory (G. Akemann, J. Baik, P. Di Francesco, eds). Oxford Univ. Press 2011, arXiv:1104.1266.

[14] S. Sheffield. Gaussian free fields for mathematicians. Probability Theory and Related Fields 139 (2007), 521-541, arXiv:math/0312099. 